\title{
INVESTIGATIONS ON PERFORMANCE CHARACTERISTICS OF FBMC: A PHY LAYER FOR NEXT GENERATION TELECOM
}

\author{
R.S.S. Maukthika, P. Namratha and P. Sri Hari \\ Department of Electronics and Communication Engineering, VNR Vignana Jyothi Institute of Engineering and Technology, India
}

\begin{abstract}
Filter bank multicarrier (FBMC) is one of the proposed physical layers for $5 G$, which promises to enable future technologies like cognitive radio, robotic automation, virtual reality etc. This paper highlights how FBMC outperforms orthogonal frequency division multiplexing $(O F D M)$ in various aspects such as data rate, spectral efficiency etc. Moreover, it provides a detailed mathematical analysis of polyphase decomposition of filter banks in FBMC and throws light on significance of offset quadrature amplitude modulation (OQAM) in dealing with channel impairments. The significance of massive MIMO systems along with the suitability of FBMC in its application has also been addressed.
\end{abstract}

Keywords:

FBMC, OFDM, Polyphase Decomposition, OQAM, Massive MIMO

\section{INTRODUCTION}

Cyclic Prefix - OFDM (CP-OFDM) has been very popular multicarrier modulation (MCM) technique in 4G-LTE systems. In a multicarrier system the channel is divided into multiple subchannels which are almost flat fading and a simple equalizer can be effectively implemented to reduce the distortion caused by wireless channel. OFDM system transmits data on set of closely spaced orthogonal 'Sinc' shaped sub-carriers for providing better spectral efficiency. To maintain orthogonality perfect synchronization of the sub-carriers is required, failing which the signal incurs heavy inter carrier interference (ICI). Hence, OFDM system undergoes significant drop in performance in Doppler spread scenarios [1] and due to requirement of perfect synchronization, flexible dynamic spectrum allocation cannot be achieved [2]. The overlapping among adjacent multicarrier symbols can be avoided by inserting a guard interval between them. But in CP-OFDM systems instead of guard interval tail of multicarrier symbol is appended at its beginning called $\mathrm{CP}$; for converting frequency selective channel into flat fading channel which makes equalization simpler. The $\mathrm{CP}$ is completely redundant is wastage of channel resource and unaffordable with the requirements of high data rate.

FBMC, an MCM technique, has been under study as a potential alternative to CP-OFDM for past decade [3] [4]. In contrast to OFDM which uses rectangular pulse with abrupt transition, the pulse shapes in FBMC decays slowly. The out of band emission is significantly suppressed by using sophisticated pulse shaping filters on each sub-channel; and hence spectral leakage in FBMC systems is decreased which reduces the impact of Doppler spread or CFO [1]. To fully exploit the channel bandwidth an overlap between adjacent sub-channels is allowed and the interference thus caused is prevented using OQAM [5] by maintaining orthogonality between them. OQAM is a special case of Staggered Multi-Tone (SMT) modulation. SMT introduces a phase shift of $\pi / 2$ between adjacent sub-channels thereby maintaining orthogonality [5]. Also, in FBMC systems, inter symbol interference (ISI) is collectively dealt by OQAM and sophisticated equalization techniques; hence no guard interval or $\mathrm{CP}$ is required [4]; facilitating effective utilization of resources. Thus FBMC proves to be one of the most preferred physical layer alternatives for 5G [4] by providing higher mobility, better spectral efficiency and lower latency compared to CP-OFDM.

\section{LITERATURE REVIEW}

Some of the early works on multicarrier modulation techniques claim that FBMC offers better performance in terms of spectral efficiency, data rate and resilience to carrier frequency offset (CFO). The detailed architecture of OFDM system, its major advantages and disadvantages are listed in [6]. In this document, use of OFDM technology in air interface in DVB-H, a digital TV standard is also brought into light. The problem of high Peak-to-average power ratio and CFO estimation and reduction are addressed in [7]. In [3] various aspects of multicarrier modulation system are presented and analyzes improved performance of FBMC over OFDM due to pulse shaping. Their work proposes an efficient pulse shaping filter popularly known as PHYDYAS filter.

FBMC-OQAM has gathered great interest of researchers in recent past. The method used in [2] - [5] describe OQAM as a powerful tool in reducing ISI and adjacent channel interference without the need for CP. Detailed implementation block diagrams of OQAM transmitter and receiver are also provided in [2]. Mathematical analysis of (Staggered Multi-Tone) SMT is presented in [1] and [5] of which OQAM is a special case. The work in [8] demonstrates that FBMC-OQAM systems can obtain better spectral efficiency and shortened length of signal burst by sending virtual symbols at the beginning and end of each data packet. An optimization algorithm is also presented in [8] for the appropriate choice of virtual symbols. Various physical layer technologies like OFDM and FBMC are studied in [4] and their respective advantages and disadvantages are highlighted. Implementation of filter banks by using sub-band coding, tree structures, lattice structures and polyphase structures can be found in [9], [10]. Transmultiplexers and their implementation using polyphase structures is presented with great detail in [11], this text also brings out the merits and demerits of polyphase structures and lattice structures which helps us to make a better choice for filter bank structures in FBMC. Interesting properties of circularly pulse shaped FBMC-OQAM systems are studied in [12] which proposes easy demodulation at the receiver by processing only either real or imaginary part of the received data without incurring any loss of information.

A clear comparison between OFDM and FBMC based on BER performance and packet delivery ratio (PDR) is presented in 
[13]. Backward compatibility of FBMC to OFDM is also mentioned as one of its advantages in [3] and [4]. In [13], the author suggests that FBMC-MIMO systems are less flexible. It is also stated in [4] that highly complex equalization is required in FBMC MIMO systems. However, [14] and [15] claims that as the order of MIMO system increases, the channel response becomes nearly flat over each sub-channel, which is called selfequalization in [14]; this ensures efficient implementation of massive MIMO systems using FBMC. FBMC has been presented as an efficient physical layer for $5 \mathrm{G}$ in [13]. It also addresses major challenges in FBMC systems such as loss of orthogonalization due to time dispersion and dynamic choice of pulse shape and adaptive transceivers for the same. Linearly processed FBMC systems are proposed in [17] which offer improved BER performance and better frequency localization (reduced OOB emission) compared to conventional FBMC systems. In [15], the bit error rate performance has been compared mathematically for OFDM and FBMC systems in doubly dispersive channels. Since OFDM does not cater to the needs of $5 \mathrm{G}$ cellular systems completely, the challenges are addressed by investigating other efficient multicarrier modulation (MCM) techniques such as FBMC.

\section{POLYPHASE IMPLEMENTATION OF TRANSMULTIPLEXERS}

In FBMC systems, MCM and sub-channel processing can be achieved using transmultiplexer (TMUX) structures [11]. The transmitter and receiver in TMUX structure consist of synthesis filter bank (SFB) and analysis filter bank (AFB) respectively. The data symbols are divided into $N$ parallel streams and modulated onto different sub-carriers. The spectrum of data on each subchannel is shrunk by a factor $N$ using up-sampling operation and passed through a pulse shaping filter with smooth roll off. The AFB does the reverse. The basic TMUX structure is shown in the Fig.1.

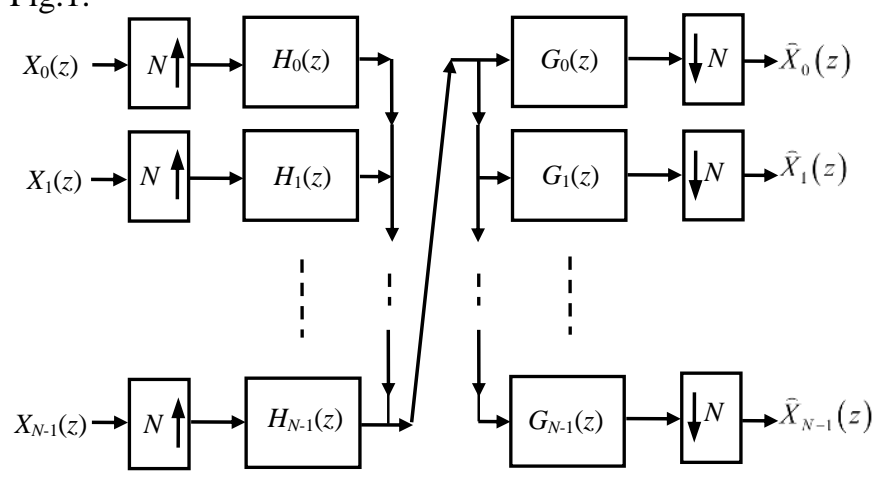

Fig.1. TMUX Structure

Let $X_{k}(z)$ be the input to the $k^{\text {th }}$ sub-channel and $H_{k}(z)$ be the $k^{\text {th }}$ sub-channel filter of SFB. Mathematically, the output of SFB is,

$$
Y(z)=\sum_{k=0}^{N-1} X_{k}\left(z^{N}\right) H_{k}(z)
$$

Instead of designing separate filters for each sub-channel, a single prototype filter can be designed and its modulated versions can be used on corresponding sub-channels. Here, $H_{k}(z)$ can be written in terms of prototype filter $H(z)$ as [6],

$$
H_{k}(z)=H\left(z W_{N}^{k}\right)
$$

where, $W$ represents the twiddle factor. Now the prototype filter and its polyphase decomposition are given by Eq.(3) and Eq.(4) respectively,

$$
\begin{aligned}
& H(z)=\sum_{n=0}^{K N-1} h(n) z^{-n} \\
& H(z)=\sum_{l=0}^{N-1} F_{l}\left(z^{N}\right) z^{-l}
\end{aligned}
$$

Here $h(n)$ is the impulse response, which can be decomposed into $N$ polyphase components each of length $K . F_{l}\left(z^{N}\right)$, the $l^{\text {th }}$ polyphase component is given by,

$$
F_{l}\left(z^{N}\right)=\sum_{m=0}^{K-1} h(m N+l) z^{-m N}
$$

Thus the polyphase equivalent of the $k^{\text {th }}$ sub-channel filter is,

$$
H_{k}(z)=\sum_{l=0}^{N-1} F_{l}\left(z^{N}\right) z^{-l} W_{N}^{-k l}
$$

The following shows the matrix representation of SFB [2].

Here,

$$
H(z)=W F\left(z^{N}\right) C(z)
$$

$$
\begin{gathered}
H(z)=\left[H_{0}(z), H_{1}(z), \ldots, H_{N-1}(z)\right]^{\mathrm{T}} \\
W=\left[\begin{array}{cccc}
W_{N}^{0} & W_{N}^{0} & \ldots & W_{N}^{0} \\
W_{N}^{0} & W_{N}^{-1} & \ldots & W_{N}^{-(N-1)} \\
\vdots & \vdots & \ddots & \vdots \\
W_{N}^{0} & W_{N}^{-N} & \ldots & W_{N}^{-N(N-1)}
\end{array}\right] \\
F\left(z^{N}\right)=\left[\begin{array}{cccc}
F_{0}\left(z^{N}\right) & 0 & \ldots & 0 \\
0 & F_{1}\left(z^{N}\right) & \ldots & 0 \\
\vdots & \vdots & \ddots & \vdots \\
0 & 0 & \ldots & F_{N-1}\left(z^{N}\right)
\end{array}\right] \\
C(z)=\left[\begin{array}{llll}
1 & z^{-1} & z^{-(N-1)}
\end{array}\right]
\end{gathered}
$$

The analysis of AFB is similar to SFB, and yield the following matrix representation,

$$
G(z)=W E\left(z^{N}\right) C(z)
$$

Here $G(z)$ is the column vector consisting of sub-channel filters of AFB, $\left.\left.G_{0}(z), G_{1}(z), \ldots, G_{N-1}(z)\right)\right)$ and $E\left(z^{N}\right)$ is the diagonal matrix consisting of polyphase components corresponding to subchannel filters of AFB as shown below,

$$
E\left(z^{N}\right)=\left[\begin{array}{cccc}
E_{0}\left(z^{N}\right) & 0 & \ldots & 0 \\
0 & E_{1}\left(z^{N}\right) & \ldots & 0 \\
\vdots & \vdots & \ddots & \vdots \\
0 & 0 & \ldots & E_{N-1}\left(z^{N}\right)
\end{array}\right]
$$

The outputs of SFB and AFB are,

$$
\begin{gathered}
Y(\mathrm{z})=C(z) F\left(z^{N}\right) \mathrm{WX}\left(z^{N}\right) \\
\widehat{X}(z)=W E\left(z^{N}\right) C(z) Y(z)
\end{gathered}
$$

where, $X(z)$ and $\hat{X}(z)$ are column vectors of sub-channel inputs of SFB and outputs of AFB respectively. The Eq.(9) and Eq.(10) represent the polyphase decomposition of transmultiplexer. By using multirate identity [9] up-samplers can be placed after subchannel filters in SFB and down-samplers can be placed before sub-channel filters in AFB. Hence the samples that are to be processed by the filters reduce by a factor $N$ [11]. 
To improve the spectral efficiency, adjacent sub-channels are allowed to overlap without causing interference which can be achieved by using OQAM. Since the sub-channels occupy double the bandwidth, maximally decimated filter bank can be obtained by reducing the up-sampling factor to half (i.e. N/2) [2]. Note that the polyphase decomposition of AFB consists of IDFT, but to achieve backward compatibility with OFDM, its DFT implementation can be achieved by simple manipulations. This results in up-side down flipping of the sub-channel filters and delay line along with addition of multiplier $W_{N}^{k}$ at the output of each sub-channel [2].

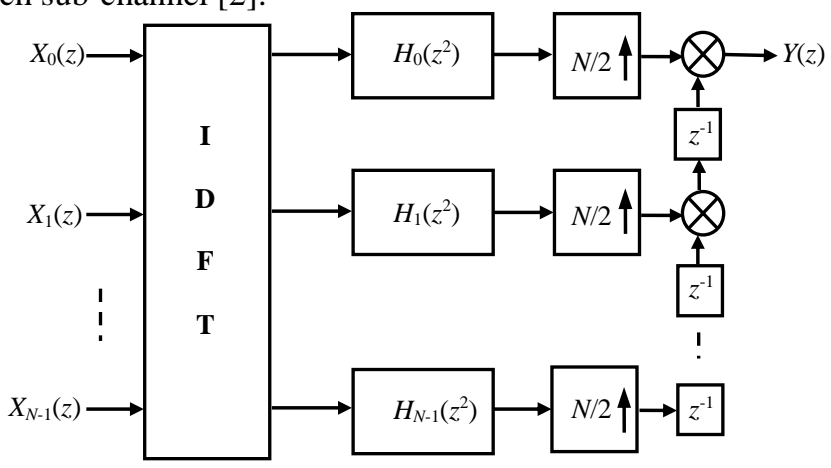

Fig.2. Polyphase decomposition of SFB

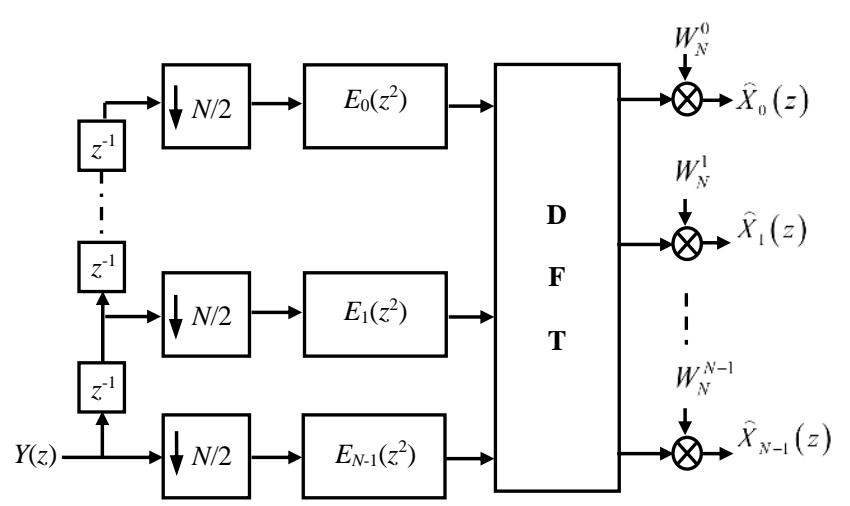

Fig.3. Polyphase decomposition of AFB

The Fig. 2 and Fig. 3 show the final polyphase implementation of transmultiplexers. To recover the transmitted signals accurately the condition $\hat{X}(z)=X(z)$ should be satisfied [10]. This is known as perfect reconstruction (PR) condition. The implementation of perfect reconstruction filter banks is more flexible using lattice structures but the cost is paid in terms of computational complexity and memory requirement [11]. By allowing some relaxation in PR condition, near perfect reconstruction (NPR) filter banks can be effectively implemented by polyphase structures using DFT/IDFT and provide higher data rate [11]. The minute errors caused by relaxation in constraints can be tolerated since they are small as compared to distortions caused by channel [2]. Thus polyphase structures prove to be more effective when high data rate is desired.

To reduce the spectral leakage beyond adjacent sub-channels, filters in FBMC must be sufficiently compressed. This results in spreading of the pulse in time domain by a factor $K$ and $K$ refers to overlapping factor [3]. Choice of prototype filter with optimal $K$ value and the design of filter bank structure are the key aspects that determine the speed and efficiency of FBMC systems.

\section{PULSE SHAPES FOR FBMC}

The main advantage of FBMC over OFDM is better spectral efficiency obtained by choosing appropriate pulse shapes. OFDM uses rectangular pulse which has abrupt transitions in time domain and side lobes in its 'Sinc' shaped spectra contain significant amount of power leading to inefficiency of spectrum utilization [3]. When all the subcarriers in OFDM are perfectly orthogonal, the peak of one subcarrier coincides exactly with the nulls of other subcarriers hence there is no ICI. But, perfect orthogonality cannot be expected in real time wireless channel which undergoes Doppler spread when the communicating terminals are in relative motion. Even small amount of resultant CFO may lead to large distortion in the signal.

The severity of ICI can be decreased by reducing the power in side lobes. Filter banks in FBMC systems are designed using a smoothly decaying pulse prototype filter which leads to significant decrease in out of band emission (OOB). There are various pulse shapes which offer good performance such as, Raised cosine pulse, Square root raised cosine pulse, IOTA (Isotropic Orthogonal Transform Algorithm), PHYDYAS filter, Hermite filter, Kalman filter etc. The choice of pulse shape can be made by comparing the power spectral densities (PSD) of the various pulse shaping filters. The Fig.4 shows the power spectral density of rectangular pulse and square root raised cosine filters with roll off factor of 0.55 and overlapping factor $K=4$. It is clear that the OOB emission is very high in OFDM.

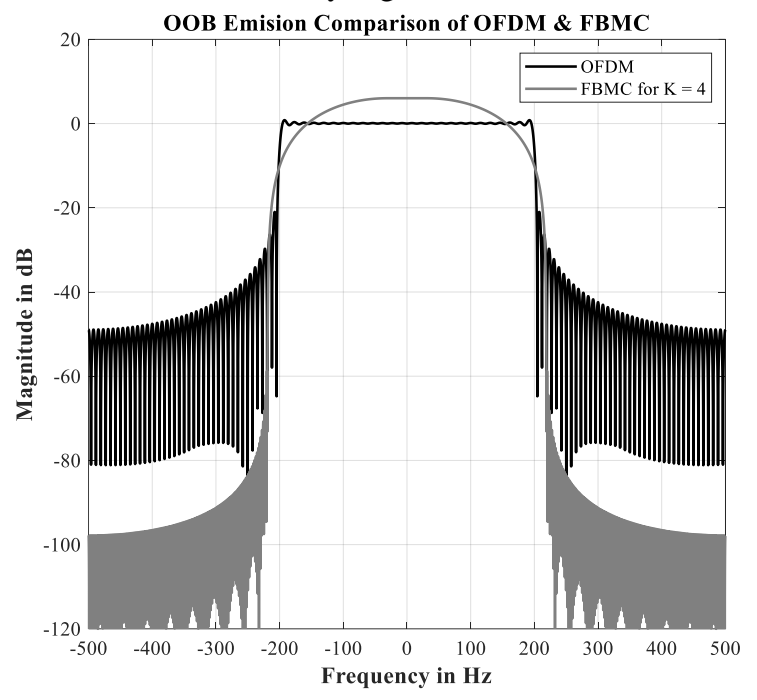

Fig.4. Comparison of Out of Band Emission in OFDM and FBMC Pulse shaping Filters

Further in Fig.5, the PSD plots of square root raised cosine pulses with different roll off factors and overlapping factors (denoted by $R$ and $K$ respectively) are considered for comparison. It is observed that as the overlapping factor increases, OOB emission decays faster. $K$ cannot be very high since it leads to longer symbol duration which is not desirable in burst communication [3], hence in most of the works $K$ value is found to be 3-4. The optimal choice of pulse shape and overlapping factor maximizes the spectral efficiency of FBMC system and effect of ICI can be reduced significantly by suppressing the PSD beyond adjacent sub-channel to negligible levels; this provides robustness to FBMC Systems in Doppler spread scenarios. 


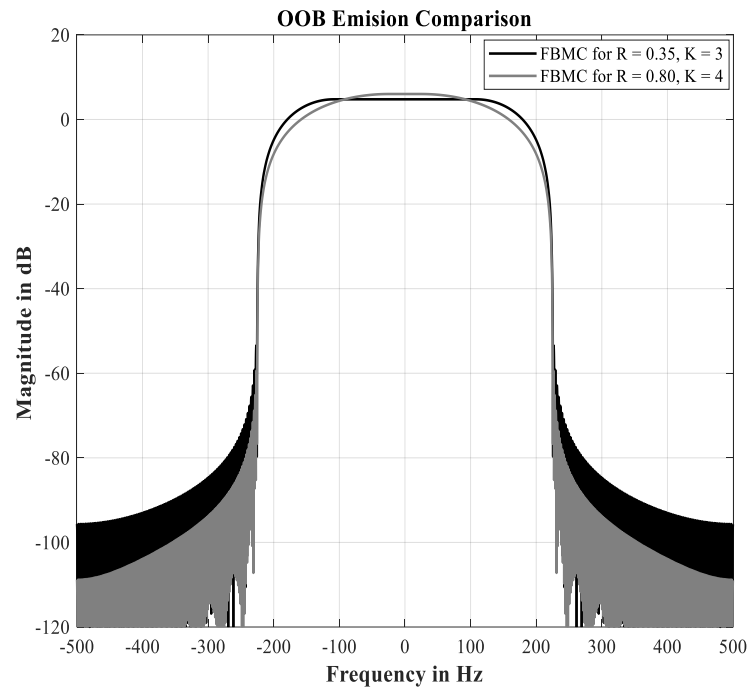

Fig.5. Comparison of Out of Band Emission for Square Root Raised Cosine Pulse with different overlapping Factors

\section{FBMC IN DOUBLY DISPERSIVE CHANNEL}

In multipath propagation scenario, dispersion in time domain causes ISI. It can be within the block (multicarrier symbol) or with other blocks. These are called intra block interference and inter block interference respectively. Using OQAM, sub-carriers carry real (imaginary) data in half the symbol duration and imaginary (real) data in the next half of the symbol duration [3]. This pattern is reversed for every multicarrier symbol which prevents inter block interference while intra block interference has to be dealt using equalization. Along with this, the choice of pulse shape also determines the extent of ISI [4] with the variation in overlapping factor $K$, which is the measure of frequency spectrum localization. By increasing $K$, maximum signal power concentrates around the centre of sub-channel and effect of frequency selectivity decreases thereby reducing ISI.

The signal reconstruction becomes even more challenging when the transmitter and receiver are in relative motion with each other; this incurs change in the channel impulse response with respect to time causing frequency dispersion. In FBMC-OQAM systems since the carrier power is negligible beyond adjacent subchannel; Doppler spread does not cause much ICI to the nonadjacent sub-channels. The extent of ICI is further reduced due to adjacent sub-channel orthogonality.

\section{FBMC IN MASSIVE MIMO SYSTEMS}

To meet the requirements of $5 \mathrm{G}$ with limited bandwidth resources, there is growing interest in massive MIMO. Interestingly, as the number of antennas increases more and more, the processing gain tends to infinite and comparatively the effect of noise on various frequency components is negligible [14]. It is a special property of massive MIMO, known as self-equalization [14] which results in nearly flat gain over each sub-channel. Hence, in FBMC massive MIMO, effect of frequency selectivity is less even though the number of sub-channels is decreased. Because of this, implementation complexity decreases significantly [15]. Moreover, as the sub-channel bandwidth increases, symbol duration decreases which results in lower latency [14]. This contributes to significant improvement in performance of FBMC massive MIMO systems.

\section{CONCLUSIONS}

FBMC systems emerge as an alternative to CP-OFDM by improving spectral efficiency, reducing sensitivity to carrier frequency offset (CFO) and enabling dynamic spectrum allocation. The mathematical analysis of transmultiplexers arrives at polyphase NPR filter banks that are computationally efficient than lattice structures, which in combination with OQAM further improves the spectral efficiency. Massive MIMO implementation in FBMC comes with added advantages; since it offers significant decrease in latency and implementation complexity. Because of all these advantages, FBMC has turned up as one of the most preferred choices for $5 \mathrm{G}$.

\section{REFERENCES}

[1] Helmut Bolcskei, Pierre Duhamel and Rima Hleiss, "Orthogonalization of OFDM/OQAM Pulse Shaping Filters Using the Discrete Zak Transform”, Signal Processing, Vol. 83, No. 7, pp. 1379-1391, 2003.

[2] Ari Viholainen, Maurice Bellanger and Mathieu Huchard, "Prototype Filter and Structure Optimization", PHYDYAS: A Physical Layer for Dynamic Access and Cognitive Radio, Technical Report, 2009.

[3] M. Bellanger, "FBMC Physical Layer: A Primer", PHYDYAS: A Physical Layer for Dynamic Access and Cognitive Radio, Technical Report, 2010.

[4] Fei Hu, "Opportunities in 5G Networks: A Research and Development Perspective", CRC Press, 2016.

[5] Sayeed Afrasiabi-Gorgani, "An Introduction to OFDMOQAM", Master Thesis, Department of Computer Science, Cornell University, 2017.

[6] B.R. Ballal, A. Chadha and N. Satam, "Orthogonal Frequency Division Multiplexing and its Applications", International Journal of Science and Research, Vol. 2, No. 1, pp. 325-328, 2013.

[7] Alcardo Alex Barakabitze and Md. Abbas Ali, "Behavior and Techniques for Improving Performance of OFDM Systems for Wireless Communications", International Journal of Advanced Research in Computer and Communication Engineering, Vol. 4, No. 1, pp. 111-119, 2015.

[8] R. Nissel, S. Schwarz and M. Rupp, "Filter Bank Multicarrier Modulation Schemes for Future Mobile Communications", IEEE Journal on Selected Areas in Communications, Vol. 35, No. 8, pp. 1768-1782, 2017.

[9] P.P. Vaidyanathan, "Multirate Systems and Filter Banks", Prentice Hall, 1993.

[10] N.J. Fliege, "Murtirate Digital Signal Processing", John Wiley and Sons, 1994.

[11] Roberto Cristi, "Modern Digital Signal Processing”, Nelson Engineering, 2004.

[12] Ahmad Rezazadeh Reyhani and Behrouz FarhangBoroujeny, "An Analytical Study of Circularly PulseShaped FBMC-OQAM Waveforms", IEEE Signal Processing Letters, Vol. 24, No. 10, pp. 1503-1506, 2017. 
[13] Bidyalaxmi Devi Tensubam, Nongmaithem Lalleima Chanu and Sonika Singh, "Comparative Analysis of FBMC and OFDM Multicarrier Techniques for Wireless Communication Networks," International Journal of Computer Applications, Vol. 100, No. 1, pp. 1317-1328, 2014.

[14] A. Farhang, N. Marchetti, L.E. Doyle and B. FarhangBoroujeny, "Filter Bank Multicarrier for Massive MIMO", Proceedings of IEEE $80^{\text {th }}$ Vehicular Technology Conference, pp. 1-7, 2014.

[15] Behrouz Farhang-Boroujeny, "Filter Bank Multicarrier Modulation: A Waveform Candidate for 5G and Beyond", Advances in Electrical Engineering, Vol. 2014, pp. 1-25, 2014.
[16] Malte Schellmann, Zhao Zhao, Hao Lin, Pierre Siohan, Nandana Rajatheva, Volker Luecken and Aamir Ishaque, "FBMC based air interface for 5G Mobile: Challenges and Proposed Solutions", Proceedings of $9^{\text {th }}$ International Conference on Cognitive Radio Oriented Wireless Networks and Communications, pp. 102-107, 2014.

[17] Jintae Kim, Yosub Park, Sungwoo Weon, Jinkyo Jeyong, Sooyong Choi and Daesik Hong, "A New Filter-Bank Multicarrier System: The Linearly Processed FBMC System", IEEE Transactions on Wireless Communications, Vol. 17, No. 7, pp. 62-74, 2018.

[18] R. Nissel and M. Rupp, "OFDM and FBMC-OQAM in Doubly-Selective Channels: Calculating the Bit Error Probability", IEEE Communications Letters, Vol. 21, No. 6, pp. 1297-1300, 2017. 